\title{
Path Analysis on Influential Factors of Somatization Symptoms in High School Girls
}

\author{
Jung-hee Jeon \\ Dept. of Nursing, Tongmyong University, \\ 428 Sinseon-ro, Nam-Gu, Busan, Korea \\ jjh@tu.ac.kr
}

\begin{abstract}
This study aims to examine the fitness of a path model that explains the relationship among factors affecting somatization symptoms in high school girls. The participants were 524 girls from 3 high schools in B city from 25, July to 30, August, 2012. The data were collected through self-reported questionnaires and analyzed by using PASW 18.0 and AMOS 16.0 program. The overall fitness indices of hypothetical model were good $\left(\chi^{2}=.03(p=.867), \chi^{2} / d f=.03, S R M R=.002, G F I=1.00, R M S E A=.00(.00-.06), N F I=1.00\right.$, $T L I=1.00, C F I=1.00)$. Out of 9 paths, 8 were statistically significant. Somatization in high school girls was directly affected by depression, academic stress, and perfectionism, except social support. This model explained $34 \%$ of variance in somatization in high school girls. The findings of this study suggest that nursing intervention to reduce somatization in high school girls should contain strategies to control their depression, academic stress, and perfectionism.
\end{abstract}

Keywords: Somatization, High school girls, Path analysis

\section{Introduction}

\subsection{Need for Study}

Somatization symptom is a psychological disorder that causes various pains, such as continuous headache, indigestion, stomachache, menstrual pain, and other physical discomfort. It is difficult to approach and mediate the actual problem of this disorder as individuals express their emotional difficulties through physical symptoms [1]. The major somatization symptoms that adolescents are complaining about are headache, dizziness, abdominal inflation, stomachache, exhausted eyes, etc. [2]. It is reported that $18.3 \%$ of adolescents who express pains like headache and stomachache suffer from frequent pain more than once a week [3]. The experience of somatization symptoms increased as one advanced to higher grades [4], and it was found that the difference between genders is most distinct during adolescence, as female students remarkably experienced more symptoms than male students $[2,4,5]$.

Adolescents showing somatization symptoms may be isolated from their peers, avoid social relationships, or show problems in social functioning, such as skipping school or performing poorly in academics [3, 6]. Moreover, there are high risks that these adolescents may habitually take excessive medication without a clear understanding and mediation of somatization [7], as well as possibilities of problems in their daily life, social and emotional maladjustment [8], and development into somatization disorder in adults [9] when somatization is not treated properly. According to DSM-IV-TR (American Psychiatric Association, 2000), somatization disorder usually occurs in teenagers and must be discovered and treated actively in the early stage, as it may continue in one's lifetime when it is not treated.

Some of the psychosocial factors related to adolescents' somatization symptoms are 
depression or anxiety, hardship during childhood, stress from school, perfectionism, and social support $[4,5,10-12]$. Specifically, stress is a factor that influences the cause and duration of somatization symptoms in adolescents, and triggers physiological somatization symptoms such as, headache, stomachache, indigestion, and emotional symptoms such as, anxiety and depression [13]. The higher the stress, the more the adolescents complained about somatization symptoms [14, 15]. Adolescence is a period with a large amount of stress due to dramatic physical, psychological, and social changes, and adolescents of higher grades had a larger amount of stress in Korea. 7 out of 10 adolescent girls were stressed from their school life, and academic stress was most heavily weighted, taking $50.4 \%$ [16] of the total stress that they experienced. The higher the academic stress, the more the adolescent girls experienced somatization symptoms [17]. Depression can be expressed as an emotional response to stress, but can also act as a factor that affects somatization $[2,5,18]$, which is why the higher the level of depression, the more the expression of somatization symptoms [19]. It is reported that perfectionism is also closely related to somatization symptoms [10]. In an environment of endless competition for college admission, adolescents with perfectionism obsessively pursued perfection due to less confidence and fear of negative evaluation from others. Perfectionist adolescents also showed symptoms such as avoiding contact and leaving an emotional gap between others, which resulted in various psychological and physical maladjustment symptoms, such as depression and somatization. Social support is reported as a psychological buffer against negative feelings such as depression and stressful incidents. It was found that social support is an influential factor in adolescents' somatization as social support from friends, parents, and teachers helped the recovery of psychological balance [5].

High school girls had higher levels of academic stress, depression [15, 16], and perfectionism compared to adolescent male students [20], and also exhibited more somatization symptoms due to their vulnerability to somatization trend [2, 22]. However, it was difficult to find studies that explained somatization on these adolescents covering various factors of somatization. In addition, prior studies related to adolescents' somatization were mostly about influential factors $[4,5]$ and mediating effects of various factors on somatization [14, 17-19], and no study that explained somatization from applying a theoretical model related to stress, the main cause of somatization, could be found. This study aims to provide basic data for the prevention and mediation of somatization symptoms by investigating the cause of somatization symptoms and stress, and promoting a clear understanding of somatization in high school girls based on the Stress Process Model from Elliot and Eisdorfer [23].

\subsection{Purpose of Study}

This study aims to develop and examine a path model related to influential factors of somatization symptoms in high school girls. Detailed purpose of study is as follows:

1) Develop a hypothetical path model that explains somatization symptoms of high school girls based on the Stress Process Model [23] and prior studies.

2) Propose a path model that explains somatization symptoms in high school girls through verification of goodness-of-fit between the hypothetical path model and real data.

3) Test the significance of estimated values from the hypothetical model, and analyze the direct, indirect, and total effect.

\subsection{Conceptual Foundation and Hypothetical Model}

This study's conceptual foundation is based on the Stress Process Model [23] and prior studies. The Stress Process Model can be the foundation of forming a nursing theory of 
stress and developing a nursing intervention, as its conceptual component of response, intervention factor, and result explain the stress process through interrelationship between these components. Stress provokes psychological maladjustment such as anxiety and depression, or acts as the cause or triggering factor of various mental diseases including physical symptoms [14]. Prior studies on the contextual relationship of the factors that affect somatization of high school girls have proved that the higher academic stress [17], the higher the level of depression $[4,5,18,19]$, and that the higher the level of perfectionism [10], and the lower the social support [5] resulted in an increase in somatization symptoms. Thus, this study established a hypothetical path model by designating academic stress as the main element of stress, depression as a reaction against stress, social support as a mediating factor, and somatization as a result, in addition to perfectionism, which has a close relationship to somatization.

\section{Method of Study}

\subsection{Design of Study}

This study is a cross-sectional research that builds a hypothetical model about the somatization symptoms of high school girls and a study on covariance path analysis that verifies the goodness-of-fit and effect of the hypothetical model built upon the basis of practical data.

\subsection{Subjects and Data Collection}

Data for this study was collected from 3 general high schools in city B from July $25^{\text {th }}$, 2012 to August $30^{\text {th }}, 2012$ upon approval for data collection. The researcher asked for cooperation after explaining the purpose of this study and method of data collection to the school nurse of each school, then guaranteed anonymity and confidentiality to the subjects after explaining the purpose and method of this study. The fact that the subject may not participate at any time whenever the subject does not want to and that data from this research will not be used elsewhere other than for research purposes were guaranteed verbally and in written form. The time spent on answering the questionnaire took approximately 10-15 minutes, and a small gift was provided for students who participated in the questionnaire. A total of 700 questionnaires were distributed, and ultimately 524 were used for analysis excluding those with insufficient answers. The sample size of this study exceeded 200, which is considered an appropriate sample size when using the maximum likelihood method.

\subsection{Tool of Study}

\subsubsection{Social Support}

A social support scale of adolescents composed of a total of 27 questions developed by Lee [24] was used for measuring social support. Each question was in a Likert scale of 5 points, and higher points indicated receiving higher social support. The reliability of the tool at the time of development was Cronbach's $\alpha=.85$, and the reliability for this study was Cronbach's $\alpha=.93$.

\subsubsection{Perfectionism}

A tool developed by Seo [25] after verification of validity against middle and high school students was used for measuring perfectionism upon approval of use by the developer. There were a total of 31 questions in a Likert scale of 5 points, and higher points indicated a higher level of perfectionism. The reliability of the tool at the time of development was Cronbach's $\alpha=.90$, and the reliability for this study was Cronbach's $\alpha=.92$. 


\subsubsection{Depression}

The CES-D (The Center for Epidemiological Studies-Depression scale) adapted by Cho and Kim [26] was used for measuring depression upon approval of use by the adapter. There were a total of 20 questions, with 1 point indicating 'extremely rare (less than a day in a week)' to 4 points indicating 'almost always.' Higher points indicated a higher level of depression. The reliability of the tool at the time of development was Cronbach's $\alpha=.85$, and the reliability for this study was Cronbach's $\alpha=.93$.

\subsubsection{Academic Stress}

The tool of academic stress of adolescents developed by Oh and Chen [27] was used to measure academic stress upon approval of use by the developer. This section was composed of a total of 42 questions, with each question in a Likert scale of 5 points. Higher points indicated a higher level of academic stress. The reliability of the tool at the time of development was Cronbach's $\alpha=.91$, and the reliability for this study was Cronbach's $\alpha=.94$.

\subsubsection{Somatization}

The Adolescent Symptom Checklist (ASC) adapted by Lee and others [28] was used in measuring somatization. This section was composed of a total of 22 questions in a Likert scale of 6 points, and higher points indicated more symptoms of somatization. The reliability of the tool in the study by Lee and others [28] was Cronbach's $\alpha=.89$, and the reliability for this study was Cronbach's $\alpha=.91$.

\subsection{Data Analysis}

Data for this study was analyzed using SPSS 18.0 and AMOS 16.0 programs.

1) SPSS 18.0 program was used to analyze the average and standard deviation of the variables, Cronbach's $\alpha$ coefficient to analyze the frequency and reliability of the general characteristics of the subjects, Pearson's Correlation Coefficient for correlation, and skewness and kurtosis for test of normality.

2) Using the AMOS 16.0 program model, the goodness of fit of the hypothesis model was evaluated by absolute fit index $\left(\chi^{2}, \chi^{2} / \mathrm{df}\right.$, SRMR [Squared Root Mean-squared Residual], GFI [Goodness-of-Fit Index], RMSEA [Root Mean Square Error of Approximation]) and incremental fit index (NFI [Normed Fit Index], TLI [TuckerLewis Index], CFI [Comparative Fit Index]). Maximum likelihood estimation was used to verify the validity of the model, as well as the validity of path coefficient and of direct, indirect, and total effect.

\section{Study Results}

\subsection{General Characteristic of Study Subjects}

The total number of subjects in this study was a total of 524 students with 219 10th graders and 30511 th graders. The average height was $161.22 \pm 5.11$ and the average body weight was $53.25 \pm 7.64$. $48.3 \%$ of the subjects answered that they regard their health condition as 'healthy,' $39.5 \%$ as 'normal,' and $12.2 \%$ as 'not so healthy.' $80.3 \%$ of the subjects answered that they normally do not exercise regularly, and $51.7 \%$ answered 'always do' to the question whether they ate breakfast regularly, $28.4 \%$ to 'mostly do,' and $10.3 \%$ to 'mostly don't,' and $9.6 \%$ to 'almost don't.' $72.1 \%$ of the subjects answered that they do not have experience in drinking alcohol, and $2.9 \%$ answered that they drink frequently, more than once a month. $92.9 \%$ of the subjects answered that they have never tried smoking, 5.0\% answered that they have smoked in the past but do not smoke now, and $2.1 \%$ that they are currently still smoking. 


\subsection{Descriptive Statistics and Correlation of Variables}

The descriptive statistics of the variables of this study were $54.01 \pm 16.95$ (range 22132) for somatization, 33.37 \pm 11.01 (range 20-80) for depression, 122.74 \pm 29.71 (range 42-210) for academic stress, 95.47 \pm 14.86 (range 27-135) for social support, and $90.14 \pm 18.22$ (range 31-155) for perfectionism (Table 1). The skewness and kurtosis test for verification of normality of the variables showed that the range of skewness was 0.18 1.26 and the range of kurtosis was $-0.31-1.14$, which satisfy the univariate normality as the absolute value of skewness was below 3.0 and the absolute value of kurtosis did not exceed 8.0.

Table 1. Descriptive Statistics if Observed Variables

\begin{tabular}{lrrrrcc}
\hline Variables & Range & Min & Max & \multicolumn{1}{c}{ M $\pm S D$} & Skewness & Kurtosis \\
\hline Depression & 52.0 & 20.0 & 72.0 & $33.37 \pm 11.01$ & 1.26 & 1.14 \\
Social support & 102.0 & 33.0 & 135.0 & $95.47 \pm 14.86$ & -0.12 & 0.71 \\
Academic stress & 164.0 & 43.0 & 207.0 & $122.74 \pm 29.71$ & -0.18 & -0.31 \\
Perfectionism & 107.0 & 42.0 & 149.0 & $90.14 \pm 18.22$ & 0.08 & 0.13 \\
Somatization & 90.0 & 22.0 & 112.0 & $54.01 \pm 16.95$ & 0.50 & 0.05 \\
\hline
\end{tabular}

The analysis result of correlation between variables for validation of the hypothesis model in this study is as Table 2. All variables excluding perfectionism and social support showed significant correlation between the variables, and multicollinearity test between the variables showed that there was no problem in multicollinearity, where the highest correlation was .54 and no correlation exceeded .90 .

Table 2. Correlation of Observed Variables

$(\mathrm{N}=524)$

\begin{tabular}{lccccc}
\hline Depression & $\begin{array}{c}\text { Social } \\
\text { support }\end{array}$ & $\begin{array}{c}\text { Academic } \\
\text { stress }\end{array}$ & Perfectionism & Somatization \\
\hline Depression & 1.00 & & & & \\
\hline Social support & $\begin{array}{c}-.40 \\
(<.001)\end{array}$ & 1.00 & & & \\
\hline Academic stress & $\begin{array}{c}.41 \\
(<.001)\end{array}$ & $\begin{array}{c}-.16 \\
(<.001)\end{array}$ & 1.00 & & \\
\hline Perfectionism & $\begin{array}{c}.28 \\
(<.001)\end{array}$ & $\begin{array}{c}.01 \\
(.867)\end{array}$ & $\begin{array}{c}.41 \\
(<.001)\end{array}$ & 1.00 & \\
\hline Somatization & $\begin{array}{c}.54 \\
(<.001)\end{array}$ & $\begin{array}{c}-.21 \\
(<.001)\end{array}$ & $\begin{array}{c}.34 \\
(<.001)\end{array}$ & $\begin{array}{c}.28 \\
(<.001)\end{array}$ & 1.00 \\
\hline
\end{tabular}

\subsection{Testing of Hypothetical Model}

1) Goodness-of-fit test and estimation of path-coefficient of hypothesis model

The goodness-of-fit of the hypothesis model of this study was $\chi^{2}=.03(p=.867)$, $\chi^{2} / \mathrm{df}=.03, \mathrm{SRMR}=.002, \mathrm{GFI}=1.00, \mathrm{RMSEA}=.00(.00-.06), \mathrm{NFI}=1.00, \mathrm{TLI}=1.00, \mathrm{CFI}=1.00$, which satisfy all standards of goodness-of-fit. An estimation value of the course set in the hypothetical path model of this study was calculated for verification of parameter and significance.

There were a total of 9 courses set in the path model of this study, and 8 courses excluding social support were statistically significant among the courses that head towards somatization.

Within the course parameter of the hypothesis model, courses that had significantly 
direct influence on somatization of high school girls were depression $(\beta=50, p<.001)$, perfectionism $(\beta=10, p=.009)$, and academic stress $(\beta=.09, p=.034)$, and $34 \%$ of the section of these variables explained somatization symptoms of high school girls. The courses that had direct influence on depression were social support $(\beta=35, p<.001)$, academic stress $(\beta=29, p<.001)$, and perfectionism $(\beta=17, p<.001)$, and the explanation power of depression by these variables was $31 \%$. The courses that had direct influence on academic stress were perfectionism $(\beta=41, p<.001)$ and social support $(\beta=17, p<.001)$, and $20 \%$ explained academic stress (Table 3 ).

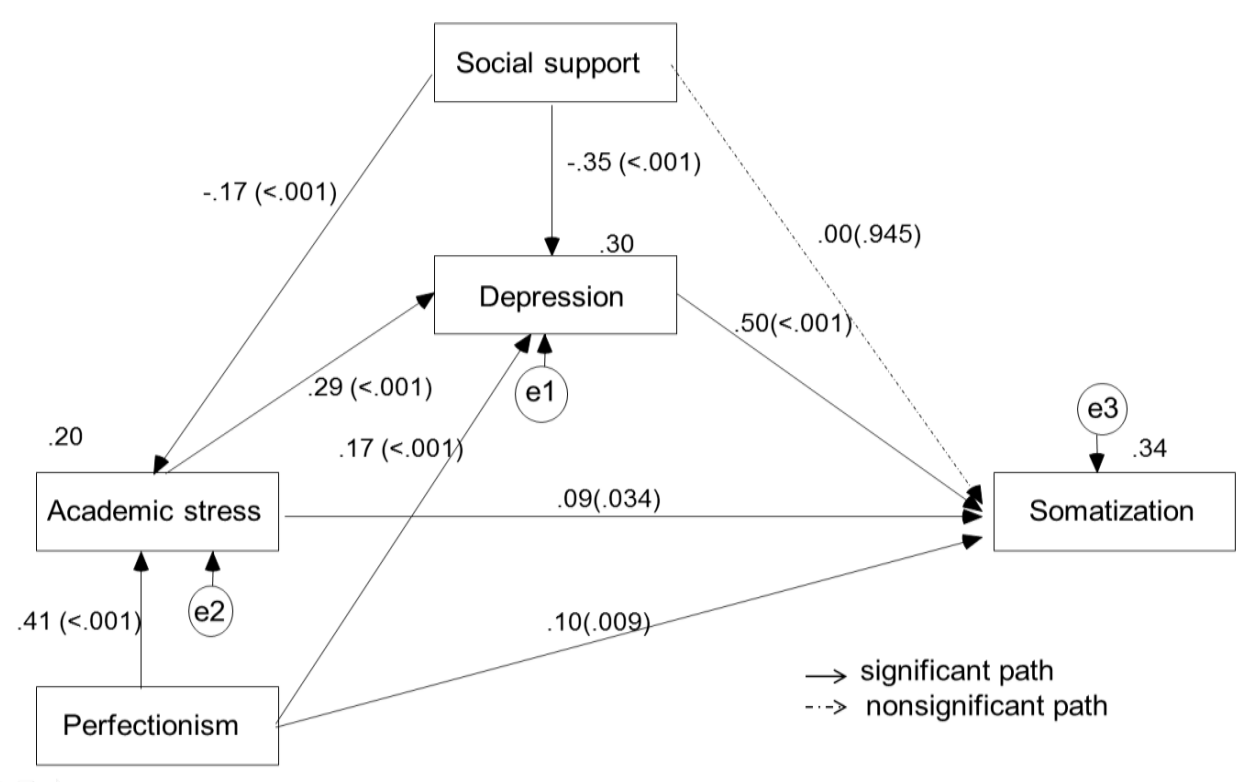

Figure 1. Hypothetical Path with Parameter Estimates

2) Effectiveness analysis of hypothesis model

An analysis of direct effect, indirect effect, and total effect of exogenous variable on endogenous variable in the hypothesis model was conducted (Table 3). The total effect of factors that affect somatization of high school girls were in the order of depression $(\beta=50$, $p=.003)$, perfectionism $(\beta=28, p=.005)$, academic stress $(\beta=23, p=.003)$, and social support $(\beta=-21, p=.004)$. Depression was the variable that had the biggest direct influence on somatization symptoms of high school girls without indirect effect, and the total effect of perfectionism $(\beta=.18, p=.003)$ and academic support $(\beta=14, p=.002)$ increased with the addition of indirect effect that was bigger than direct effect. Social support $(\beta=-21, p=.004)$ had no direct influence on the somatization of high school girls, but the total effect was significant as the indirect effect by depression and academic stress were significant.

The total effect on depression were in the order of social support $(\beta=-40, p=.007)$, academic stress $(\beta=29, p=.003)$, and perfectionism $(\beta=29, p=.005)$, and in the order of perfectionism $(\beta=41, p=.003)$ and social support $(\beta=17, p=.003)$ for academic stress. 
Table 3. Effects of Variables in the Hypothetical Model

\begin{tabular}{|c|c|c|c|c|c|c|c|c|c|c|}
\hline $\begin{array}{l}\text { Endogenous } \\
\text { variables }\end{array}$ & $\begin{array}{l}\text { Exogenous } \\
\text { variables }\end{array}$ & $B$ & SE & $\beta$ & C.R. & $p$ & SMC & Direct & Indirect & Total \\
\hline \multirow[t]{4}{*}{ Somatization } & Social support & -.00 & .05 & -.00 & -.07 & .945 & .34 & $\begin{array}{l}-.00 \\
(.945)\end{array}$ & $\begin{array}{l}-.21 \\
(.004)\end{array}$ & $\begin{array}{c}-.21 \\
(.004)\end{array}$ \\
\hline & Depression & .77 & .07 & .50 & 11.68 & $<.001$ & & $\begin{array}{c}.50 \\
(<.001)\end{array}$ & & $\begin{array}{c}.50 \\
(.003)\end{array}$ \\
\hline & Academic stress & .05 & .02 & .09 & 2.13 & .034 & & $\begin{array}{l}.09 \\
(.034)\end{array}$ & $\begin{array}{l}.14 \\
(.002)\end{array}$ & $\begin{array}{c}.23 \\
(.003)\end{array}$ \\
\hline & Perfectionism & .10 & .04 & .10 & 2.60 & .009 & & $\begin{array}{l}.10 \\
(.009)\end{array}$ & $\begin{array}{l}.18 \\
(.003)\end{array}$ & $\begin{array}{l}.28 \\
(.005)\end{array}$ \\
\hline \multirow[t]{3}{*}{ Depression } & Social support & -.26 & .03 & -.35 & -9.42 & $<.001$ & .31 & $\begin{array}{c}-.35 \\
(<.001)\end{array}$ & $\begin{array}{l}-.05 \\
(.002)\end{array}$ & $\begin{array}{c}-.40 \\
(.007)\end{array}$ \\
\hline & Academic stress & .11 & .02 & .29 & 7.07 & $<.001$ & & $\begin{array}{c}.29 \\
(<.001)\end{array}$ & & $\begin{array}{c}.29 \\
(.003)\end{array}$ \\
\hline & Perfectionism & .10 & .02 & .17 & 4.11 & $<.001$ & & $\begin{array}{c}.17 \\
(<.001)\end{array}$ & $\begin{array}{c}.12 \\
(.003)\end{array}$ & $\begin{array}{c}.29 \\
(.005)\end{array}$ \\
\hline \multirow[t]{2}{*}{$\begin{array}{l}\text { Academic } \\
\text { stress }\end{array}$} & Social support & -.33 & .08 & -.17 & -4.22 & $<.001$ & .20 & $\begin{array}{l}-.17 \\
(<.001)\end{array}$ & & $\begin{array}{c}-.17 \\
(.003)\end{array}$ \\
\hline & Perfectionism & .67 & .06 & .41 & 10.55 & $<.001$ & & $\begin{array}{c}.41 \\
(<.001)\end{array}$ & & $\begin{array}{c}.41 \\
(.003)\end{array}$ \\
\hline
\end{tabular}

$\mathrm{S} . \mathrm{E}=$ Standard error; C.R.=Critical Ratio; $\mathrm{SMC}=$ Squared Multiple Correlation.

\section{Discussion}

This study has set the courses of academic stress, depression, social support, and perfectionism that influence somatization of high school girls based on the Stress Process Model by Elliot and Eisdorfer [23] and prior studies, established a hypothetical model of somatization in high school girls, and verified the goodness-of-fit of the model and significance of the courses. Discussion based on the major results of this study is as follows:

Factors that had direct influence on somatization symptoms of high school girls were academic stress, depression, and perfectionism, and 34\% of them explained by somatization symptoms.

In this study, depression was shown to have the largest direct effect on the somatization of high school girls, and the higher the level of depression, the more they complained of somatization symptoms. This result corresponded with the results of prior studies on the influence of depression on somatization symptoms [5, 9, 18, 19, 21, 29]. Adolescent depression is a developmental characteristic, which increases dramatically compared to childhood and is expressed in a concealed form of depression unlike adults. Thus, even a minor somatization symptom may intensify the experience of somatization symptoms during a depressive state as it influences the attribution of the causes of somatization [12] compared to when in a positive or neutral state. It is also correlated to various somatization symptoms such as fatigue, hypersomnia, weight gain, etc., [30]. Moreover, in a prior study that investigated the emotional factors that influence somatization symptoms by the difference of development [4], somatization of elementary and middle school students were affected by anxiety, high school students by depression, and college students by depression and anxiety. Comparing these results, this study has shown that depression causes an effect on the somatization of high school girls, which allows for understanding in the same context of the results of prior studies. Based upon these results, we can conjecture that different emotional factors may influence somatization symptoms at different stages of development, and there is a need of verifying emotional factors at different stages of development through repeated studies, as well as an establishment of 
intervention to somatization in the future. In this study, depression showed an increasing trend when academic stress and perfectionism increased and social support decreased. This corresponds to the results of prior studies that showed that depression was induced [13] by stress reaction, and that the level of depression increased as the amount of stress increased [31], perfectionism increased [32], and social support from friends, parents, and teachers decreased [33]. Thus, it is suggested that controlling the level of depression through regulating academic stress and perfectionism, as well as increasing social support, may be helpful in relieving somatization symptoms in high school girls.

The main source of stress in high school girls was academic stress, and it was proved that the higher the academic stress, the more somatization symptoms they experienced, which correspond to prior studies [17]. According to the 2014 statistical results of adolescents [16], 11.2\% of the adolescents thought of committing suicide within the past year, and $39.2 \%$ of these adolescents answered that the main reason they wanted to commit suicide was because of academic stress. One may be able to know how severely adolescents are stressed by academics as it is reported that academic stress is the most complained stress in high school students due to the competitive trend of college entrance examination and heated education environment, and that the level of stress is higher in female students than male students [16]. Despite the severity of the situation, adolescents are still lacking the time and healthy leisure activity to relieve stress properly. Female students in particular tend to internalize stress when confronting a stressful situation and express stress with somatization symptoms. Thus, the high level of academic stress was proved to have a direct effect on somatization. This study not only showed that academic stress has a direct influence on somatization, but also that it corresponds to results of prior studies [31], where an increase of academic stress causes an increase in the level of depression, which eventually causes an increase in the effect on somatization in addition to the direct effect mediated by depression. Thus, it is suggested that a mediation controlling academic stress may be useful in the efficiency of mediation as it can relieve direct and indirect symptoms and display synergistic effect of mediation.

The result of this study indicated that perfectionism is the second largest factor that influences somatization in high school girls. This result corresponded with prior studies where stronger levels of perfectionism increased the complaints of somatization symptoms [10]. Perfectionism has a positive aspect in that it increases the motivation or performance level of academics or work, but it also causes people to be discouraged easily and experience maladjustment in the everyday life from setting up unreasonable goals beyond one's ability and evaluating oneself critically. Thus, it is reported that people with high levels of perfectionism show more symptoms of somatization in a situation where his/her performance is about to fail. This study proved that the need for avoiding extreme perfectionism in adolescents should not be overlooked, as stronger levels of perfectionism increased psychological maladjustment such as academic stress and depression [32] and worsened somatization symptoms [10].

Unlike the results from prior studies [5] that indicated that social support is a factor that influences the somatization symptoms of adolescents, this study showed that social support did not have a direct effect on the somatization of high school girls. However, it was shown that the total effect had a significant influence on somatization, as there was a significant direct effect of depression and academic stress as mediations. The explanation of the influence social support has on mental health can be divided into 2 types of main effect hypotheses: a stress-buffer hypothesis where support from parents, teachers, and friends play a role as a buffer from the negative school-related influence, and a hypothesis that states social support brings direct positive results to one's health, regardless of the level of stress. This study supports the stress-buffer hypothesis, as social support did not show a direct positive effect on somatization, but an indirect effect through academic stress and depression. This result can be understood in a similar context as a prior study that proved that social relationship formed by negative social interaction such as 
perceived stress and depression has a stronger and more continuous relationship than social support [34], and another study that indicated that social support did not show a significant relationship to somatization, but may be an influencing factor of somatization in adolescents [5]. Thus, it is suggested that the strategy of regulating somatization through controlling depression and stress should be preferred over a direct regulation through social support.

As the results suggest, somatization of high school girls is difficult to control through simply relieving somatization symptoms, as various factors such as depression, academic stress, and perfectionism are the reason for somatization symptoms of high school girls. Thus, a proper intervention and clear understanding of health problems similar to the complicated somatization symptoms of high school girls are required, considering the factors that influence somatization symptoms of high school girls shown in this study.

This study explained the symptoms of somatization by setting up courses of the influence academic stress, depression, social, and perfectionism have on somatization symptoms, based on the theory of the Stress Process Model. This study can be considered significant and meaningful as it suggested a direction for the prevention and regulation program of somatization in high school girls by including the results of individuallyconducted prior studies to explain the causal relationship between the factors, and by overcoming the limitation of mediations of somatization symptoms without a theoretical foundation.

\section{Acknowledgments}

This study was investigated to better understand somatization of high school girls by using the Stress Process Model by Elliot and Eisdorfer (1982) as a conceptual basis, setting up a course based on the prior studies and papers related to somatization of high school girls, establishing a hypothetical model, and verifying the hypothesis and goodness-of-fit of the model.

Data of this study was collected from 524 high school girls, and conducted establishment and verification of hypothesis model.

The goodness-of-fit of the hypothesis model was $\chi^{2}=.03(p=.867), \chi^{2} / \mathrm{df}=.03$, $\mathrm{SRMR}=.002, \mathrm{GFI}=1.00, \mathrm{RMSEA}=.00(.00-.06), \mathrm{NFI}=1.00$, TLI $=1.00, \mathrm{CFI}=1.00$, which satisfies all standards of the goodness-of-fit. Among the 9 courses set in the hypothesis model, 8 of the courses, excluding social support, that lead to somatization were statistically significant. Within the parameters of the hypothesis model, courses that had a significantly direct effect on somatization of high school girls were in the order of depression, perfectionism, and academic stress, and 34\% of section of these variables explained somatization symptoms of high school girls. Through the results of this study, it is suggested that solving negative emotions such as depression, avoiding the formation of extreme perfectionistic personality, and reducing academic stress are needed to decrease the somatization symptoms in adolescents. The results of this study will be a baseline data in developing a strategy for reducing somatization symptoms in high school girls, and is expected to be a theoretical basis for the development of intervention programs for reducing somatization symptoms in high school girls in the future.

\section{References}

[1] Z. J. Lipowski, "Somatization: The concept and its clinical application", American Journal of Psychiatry, vol. 145 , no. 11 , (1988), pp. 1358-1368.

[2] S. Y. Kim and H. Eh, "Validation study of the korean children's somatization inventory for high school students", Cognitive Behavior Therapy in Korea, vol. 9, no. 2, (2009), pp. 25-37.

[3] B. Larsson and A. M. Sund, "Emotional/behavioral, social correlates and one-year predictors of frequent pains among early adolescents: Influences of pain characteristics", European Journal of Pain, vol. 11, no. 1, (2007), pp. 57-65. 
[4] J. M. Kim and H. Jee, "A Study on the emotional, cognitive and social variables for somatization by developmental stage”, Korean Journal of Counseling, vol. 12, no. 4, (2011), pp. 1109-1130.

[5] H. J. Lee and M. A. Sae, "Factors influencing somatization in adolescents", Journal of Korean Society of School Health, vol. 23, no. 1, (2010), pp. 79-87.

[6] A. Konichezky and D. Gothelf, "Somatoform disorders in children and adolescents", Harefuah, vol. 150, no. 2, (2011), pp. 180-184.

[7] J. A. Trafton, M. A. Cucciare, E. Lewis and M. Oser, "Somatization is associated with non-adherence to opioid prescriptions", Journal of Pain, vol. 12, no. 5, (2011), pp. 573-580.

[8] S. Rajindrajith, N. M. Devanarayana, L. Weerasooriya, W. Hathagoda and M. A. Benninga, "Quality of life and somatic symptoms in children with constipation: A school-based study", The Journal of Pediatrics, vol. 163, no. 4, (2013), pp. 1069-1072.

[9] S. Fischer, J. Gaab, U. Ehlert and U. M. Nater, "Prevalence, overlap, and predictors of functional somatic syndrome in a student sample", International J. Behavioral Medicine, vol. 20, no. 2, (2013), pp. 184-193.

[10] S. H. Chung and J. Y. Lee, "The relation between perfectionism and somatization among children: The moderating effects of stress-coping behaviors", Korean Journal of Counseling, vol. 13, no. 2, (2012), pp. 1029-1042.

[11] R. A. Shannon, M. D. Bergren and A. Matthews, "Frequent visitors: Somatization in school-age children and implications for school nurses", The Journal of School Nursing, vol. 26, no. 3, (2010), pp. 169-182.

[12] H. K. Shin "Effects of depressive mood state on self-focused attention, somato-sensory amplification, somatic attribution, and somatic symptoms in somatization group", The Korean J. Clinical Psychology, vol. 25, no. 2, (2006), pp. 467-488.

[13] P. A. Thoits, "Stress and health: Major findings and policy implications", Journal of Health and Social Behavior, vol. 51, no. 5, (2010), pp. 541-553.

[14] M. G. Park and C. N. Son, "Mediating effects of worry in the relation of stress and somatization", Korean Journal of Stress Research, vol. 19, no. 4, (2011), pp. 361-369.

[15] J. Y. Ham and K. Park, "The relationships of stress, alexithymia and somatization of adolescents Focused on the high school student”, Journal of Student Guidance and Counseling, vol. 18, (2005), pp. $77-90$.

[16] "Korean Statistical Information Service", 2014 Statistics on the youth. Retrieved September 1, 2014,http;//kosis.kr/ebook, (2014).

[17] E. S. Choi, "A study on relationship between high school student's academic stress, somatization symptoms and internal-external locused control[master's thesis]", Seoul: Sogang University, (2011).

[18] Y. J. Jeong and H. Eh, "The modeling effects of the anger expression type on the relationships between adolescent's depression and somatization", The Korean J. School Psychology, vol. 11, no. 1, (2014), pp. $1-18$.

[19] S. J. Lee and Ha Eh, "The mediating effects of cognitive factor on the relationships between female adolescent's depression and somatization”, The Korean J. School Psychology, vol. 7, no. 1, (2010), pp. 55-68.

[20] Y. S. Lee and S. Y. Park, "The relationships between adolescent's maladaptive and adaptive perfectionism and worry: The moderating role of problem-solving confidence", The Korean Journal of the Human Development, vol. 13, no. 3, (2009), pp. 113-129.

[21] V. Ruchkin and M. Schwab-Stone, "A longitudinal study of somatic complaints in urban adolescence: The role of internalizing psychopathology and somatic anxiety", Journal of Youth and Adolescence, vol. 43 , no. 5, (2014), pp. 834-845.

[22] H. Rhee, D. Holditch-Davis and M. S. Miles, "Patterns of physical symptoms and relationships with psychosocial factors in adolescents", Psychosomatic Medicine, vol. 67, no. 6, (2005), pp. 1006-1012.

[23] G. R. Elliot and C. Eisdorfer, "Stress and human health: An analysis and implications of research", A study by the Institute of Medicine, National Academy of Sciences New York: Springer Publishing (1982).

[24] M. R. Lee, "A study on the social system to influence on the high school students' adaptation to school life", master's thesis, Gyeonsan; Taegu University, (2001).

[25] J. Y. Seo, "Validation of perfectionism scale for middle school and high school student [master's thesis]", Seoul; Sookmyung University, (2009).

[26] M. J. Cho and K. H. Kim, "Diagnostic validity of the CES-D (korean version) in the assesment of DSMIII-R major depression”, Journal of Neuropsychiatric Association, vol. 32, no. 3, (1993), pp. 381-399.

[27] M. H. Oh and S. M. Cheon, "Analysis of academic stressors and symptoms of juveniles and effects of meditation training on academic stress reduction", Journal of Human Understanding and Counseling, vol. 15, (1995), pp. 63-95.

[28] M. S. Lee, Y. Choi and J. S. You, "The relationship of somatic symptoms and self-esteem in adolescence", Journal of Neuropsychiatric Association, vol. 39, no. 2, (2000), pp. 323-333.

[29] J. H. Jeon, "Influencing factors of adolescent girl's somatization", Proceedings International workshop Health and Nursing 2015, Jeju Island, Korea, Advanced Science and Technology Letters (ASTL), vol. 104, (2015) August 19-22, pp. 128-133. 
[30] B. Weiss and J. Barber, "Developmental differences in the phenomenology of depression", Development and Psychopathology, vol. 15, (2003), pp. 403-430.

[31] H. S. Jwa, "The effect of academic stress on depression in adolescents: Moderating effect of gender role stereotype", Health and Social Welfare Review, vol. 34, no. 2, (2014), pp. 334-366.

[32] Y. H. Kim and S. Y. Seo, "A review of perfectionism: Assessment and treatment", The Korean Journal of Counseling and Psychotherapy, vol. 20, no. 3, (2008), pp. 581-613.

[33] Y. M. Sohn, "The effect of the adolescent's social support on their depression [master's thesis]", Suncheon; Sunchon University, (2012).

[34] J. M. Lackner, G. D. Gudleski, R. Firth, L. Keefer, D. M. Brenner, K. Guy, C. Simonetti, C. Radziwon, S. Quinton, S. S. Krasner, L. Katz, G. Garbarino, G. Iacobucci and M. D. Sitrin, "Negative aspects of close relationships are more strongly associated than supportive personal relationships with illness burden of irritable bowel syndrome", Journal of Psychosomatic Research, vol. 74, no. 6, (2013), pp. 493-500.

\section{Author}

\section{Author's Name: Jung-hee Jeon}

Author's profile: Dept. of Nursing Assistant Professor, Ph. D 
International Journal of $u-$ and e- Service, Science and Technology Vol.8, No. 11 (2015) 